\title{
AS AÇÕES DOS EMPREENDEDORES DO TIPO CAUSATION E EFFECTUATION COMO PREDITORAS DO COMPORTAMENTO EMPREENDEDOR
}

Luciano Da Costa Barzotto ${ }^{1}$

Vânia Maria Jorge Nassif ${ }^{2}$

\footnotetext{
${ }^{1}$ Universidade Nove de Julho

${ }^{2}$ Mestrado e Doutorado / Programa de Pós-Graduação em Administração / Universidade Nove de Julho
} 


\section{AS AÇÕES DOS EMPREENDEDORES DO TIPO CAUSATION E EFFECTUATION COMO PREDITORAS DO COMPORTAMENTO EMPREENDEDOR}

Resumo: O comportamento do tipo Effectuation transforma contingências em soluções possíveis, ideias em oportunidades de inovação e network em recursos essenciais para a consecução das ações empreendedoras, e o tipo Causation se reconhecem as oportunidades, se implantam detalhados planos de ação e são mensurados retornos sobre o capital investido. $\mathrm{O}$ presente estudo é de natureza teórica, cuja finalidade foi identificar as diferenças dos comportamentos do tipo Causation e Effectuation através das ações empreendedoras. Foram analisados o desenvolvimento das pesquisas de Causation e Effectuation em 36 artigos, dentre os 152 mais citados na Base de dados da Web of Science, e os resultados apontam que as diferenças de comportamento no uso das lógicas Causation e Effectuation são predominantemente contextuais, e a propensão de escolha entre elas é determinada pelas heurísticas do empreendedor que, dentro de situações específicas de risco ou incerteza, elegerá a mais adequada ou preferencial a ser adotada.

Palavras-chave: Empreendedorismo. Empreendedor. Effectuation. Causation.

\section{Introdução}

Pesquisadores que têm se dedicado aos estudos do empreendedorismo observam que há uma multiplicidade de visões e conceitos deste fenômeno nas diferentes áreas de conhecimento, o que indica pouco consenso entre as diversas abordagens (Shane \& Venkataraman, 2000; Shane, 2012).

Desta maneira, enquanto, por exemplo, os economicistas vinculam o empreendedor à inovação (Shumpeter, 1934), os comportamentalistas (McClelland, 1961) focam nas características criativas, na perseverança e na liderança. Também, os pesquisadores que estudam o processo empreendedor de criação de novas empresas veem como características dominantes o valor, a adaptabilidade, bem como as qualificações individuais como determinantes para o sucesso (Filion, 1999).

O trabalho de March (1991) e Levinthal \& March (1993) serviu de base para alguns dos estudos que discutem a mudança e a exploração de oportunidades empreendedoras, cujos conceitos de Exploration e Exploitation e as suas inter-relações colaboram nas pesquisas do empreendedorismo e também na prospecção de outra teoria do empreendedorismo, conhecida como Effectuation (Sarasvathy, 2001a, 2001b, 2008). Não por acaso, todos os constructos mencionados se originaram dos estudos acerca da Racionalidade Limitada (Simon, 1947) que, associados às pesquisas da Teoria Comportamental de Cyert \& March (1963), buscam explicar os comportamentos humanos e a tomada de decisão do empreendedor, adaptável aos vários contextos organizacionais.

Dentre as abordagens emergentes que estudam o empreendedor e seus comportamentos está a teoria Effectuation (realização, em tradução livre), a qual pode ser entendida como uma lógica

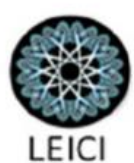


de controle de um futuro imprevisível, em sentido oposto ao Causation, que se assenta sobre bases de predição (Sarasvathy, 2001a, 2001b, 2008). Enquanto o primeiro vem a ser uma maneira diferente de compreender e desafiar o modo com que as ações empreendedoras e o comportamento do empreendedor podem ser entendidos pois fazem uso daquilo que detém posse (quem são, o que conhecem e com quem se relacionam) e fazem uma opção, entre os vários tipos de resultados possíveis, o segundo (Causation) vem alicerçado em planos formais para tentar prever o futuro.

Ao analisar a teoria proposta por March (1991), Sarasvathy (2001a) argumenta que, da mesma forma que as organizações podem adotar indistintamente o Exploitation e o Exploration, de acordo com o ambiente empreendedor, Causation e Effectuation igualmente não são excludentes entre si, e sim processos organizacionais complementares. Alternativamente, podem ser aplicados conforme seja necessário adotar esta ou aquela escolha adaptativa à realidade empreendedora que envolve risco e incerteza, em que essa incerteza caracteriza a impossibilidade de ser mensurada, dado ao futuro, que não é conhecido (Knight, 1921) e ambientes onde existe alguma dificuldade em planejar ou mesmo prever cenários (Mintzberg, 1994).

Neste aspecto, para as finalidades deste trabalho, optamos pelo campo teórico, por identificar as diferenças dos comportamentos do tipo Causation e Effectuation frente às ações empreendedoras. A relevância deste estudo está no mapeamento das pesquisas, seus métodos de pesquisa, os pontos congruentes,_além dos desafios a serem superados.

Após a introdução deste artigo, segue a revisão da literatura, abordando os temas acerca de Empreendedorismo, Causation e Effectuation, a metodologia utilizada, as discussões, seguidas das considerações finais do artigo, em que apresentamos as relações entre os constructos, bem como as contribuições.

\section{Referencial Teórico}

A literatura, de maneira geral, pontua que Exploitation se refere ao aproveitamento daquilo que já é de conhecimento dos indivíduos, cujas ideias podem ser associadas a rotinas, à clareza dos caminhos a serem percorridos e a experiências prévias que conduzem a antigas certezas e facilitada mensuração. Por outro lado, o Exploration está intimamente ligado às incertezas do novo, a caminhos que envolvem risco, à busca de novas alternativas, à flexibilidade e, portanto, implica descobertas, novas possibilidades, novos conhecimentos, aprendizado e inovação (March, 1991). Ambas, nesse sentido, envolvem a necessidade de indivíduos e de organizações realizarem uma escolha com base em suas experiências.

Sarasvathy (2001a, p.245) usa de um artifício de dicotomia para conceituar os processos Effectuation, a partir dos processos Causation, e define esses como "processos que tomam um determinado efeito como dado e locus sobre a seleção entre os meios para criar esse efeito" e aqueles - os processos de Effectuation - "como um conjunto de meios como dado e locus na seleção entre os possíveis efeitos que podem ser criados com esse conjunto de meios". Ao comparar um com o outro, Sarasvathy (2001a, 2001b) aponta que, enquanto os processos Causation, originados a partir de um paradigma teórico economicista (Kirzner, 1979, 1982) e também de processos de causa e efeito (Porter, 1980), são ótimos para se lidar com um conhecimento preexistente. Os processos Effectuation, apoiados em princípios teóricos

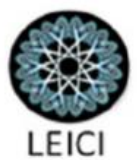


comportamentalistas, são preferíveis quando ocorrem contingências, e estas sempre estarão intrinsecamente ligadas às decisões humanas e à busca de novos conhecimentos. $\mathrm{O}$ artigo seminal de Sarasvathy (2001a) focou, inicialmente, em gerar uma teoria para explicar como as novas empresas eram criadas. Para simplificar e entender as diferenças entre as características distintivas nos Processos Causation e nos Processos Effectuation, estes estão demonstrados na Figura 1:

\begin{tabular}{|c|c|c|}
\hline $\begin{array}{l}\text { Categoria de } \\
\text { diferenciação }\end{array}$ & Processos Causation & Processos Effectuation \\
\hline Dados & Efeito é dado/fornecido & $\begin{array}{l}\text { Somente alguns meios/ferramentas são } \\
\text { dados/fornecidos }\end{array}$ \\
\hline $\begin{array}{l}\text { Critérios de seleção } \\
\text { para tomada de } \\
\text { decisão }\end{array}$ & $\begin{array}{l}\text { Ajudar a escolher entre os meios para alcançar o } \\
\text { efeito dado; } \\
\text { Critérios de seleção com base no retorno } \\
\text { esperado; } \\
\text { Efeito dependente: a escolha dos meios é } \\
\text { impulsionada pelas características do efeito que } \\
\text { o tomador de decisão quer criar e seu } \\
\text { conhecimento acerca dos meios possíveis; }\end{array}$ & $\begin{array}{l}\text { Ajude a escolher entre possíveis efeitos que } \\
\text { podem ser criados com determinados meios; } \\
\text { Critérios de seleção baseados em perda } \\
\text { acessível ou risco aceitável; } \\
\text { Ator dependente: dados meios específicos, a } \\
\text { escolha do efeito é conduzida pelas } \\
\text { características do ator e sua capacidade de } \\
\text { descobrir e usar contingências. }\end{array}$ \\
\hline $\begin{array}{l}\text { Competências } \\
\text { empregadas }\end{array}$ & Excelente na exploração do conhecimento & Excelente na exploração de contingências. \\
\hline $\begin{array}{ll}\text { Contexto } & \text { de } \\
\text { relevância } & \end{array}$ & $\begin{array}{l}\text { Mais presente na natureza; } \\
\text { Mais útil em ambientes estáticos, lineares e } \\
\text { independentes }\end{array}$ & $\begin{array}{l}\text { Mais onipresente na ação humana; } \\
\text { Premissa de ambientes dinâmicos, não } \\
\text { lineares e ecológicos. }\end{array}$ \\
\hline $\begin{array}{ll}\text { Natureza } & \text { das } \\
\text { incógnitas }\end{array}$ & $\begin{array}{l}\text { Foco nos aspectos previsíveis de um futuro } \\
\text { incerto }\end{array}$ & $\begin{array}{l}\text { Foco nos aspectos controláveis de um futuro } \\
\text { imprevisível. }\end{array}$ \\
\hline Lógica central & $\begin{array}{l}\text { Na medida em que podemos prever o futuro, } \\
\text { podemos controlá-lo }\end{array}$ & $\begin{array}{l}\text { Na medida em que possamos controlar o } \\
\text { futuro, não precisamos prevê-lo. }\end{array}$ \\
\hline Resultados & $\begin{array}{l}\text { Participação de mercado nos mercados } \\
\text { existentes através de estratégias competitivas }\end{array}$ & $\begin{array}{l}\text { Novos mercados criados através de alianças } \\
\text { e outras estratégias cooperativas }\end{array}$ \\
\hline
\end{tabular}

Figura 1 - Características distintivas entre o processo do tipo Causation e do tipo Effectuation.

Fonte: Traduzido de Sarasvathy (2001a).

A ação que leva o sujeito a empreender tem sua gênese em uma análise reflexiva pessoal acerca dos meios disponíveis, cujas perguntas são: "Quem sou eu?" (Sua identidade e suas características e habilidades), "O que eu sei? (Conhecimentos ou mesmo informações que detém)" e "Quem eu conheço?" (Stakeholders, networks ou redes sociais), permitem considerar o que este, com os meios então disponíveis, pode realizar a partir daí (Sarasvathy, 2001a, 2001b, 2008; Sarasvathy \& Dew, 2005). O raciocínio do Effectuation pode ser ainda explicado por quatro princípios, segundo Sarasvathy (2001a, 2001b, 2008): 1. Perdas toleráveis: o empreendedor estabelece um nível de perda aceitável e, mesmo com uma limitação de recursos, procura experimentar outras estratégias e julga serem possíveis; 2. Alianças estratégicas: procura viabilizar parcerias com sua rede de contatos de maneira a construir comprometimentos antecipados que possibilitem reduzir as incertezas e melhorar sua performance frente aos concorrentes; 3. Exploração de contingências: procura se aproveitar das dificuldades e contingências que podem aparecer ao longo do tempo e transformar em oportunidades, e 4. Controle de um futuro imprevisível: enquanto o Causation se baseia na expectativa de prever ou antecipar os acontecimentos, o Effectuation procura controlar o futuro tido como imprevisível. 
Percebe-se assim que a interação com os diversos stakeholders é fundamental no Effectuation, para que seja possível estabelecer, primeiramente, comprometimentos sobre o futuro e, posteriormente, acordos estratégicos, considerando que "a oportunidade é produzida por meio de um processo que transforma continuamente as realidades existentes em possíveis mercados" (Sarasvathy \& Dew, 2005 p. 544).

Sob este aspecto, ao se estudar, por exemplo, o uso da abordagem Effectuation aplicada na tomada de decisão sob incerteza, pelos empreendedores, na internacionalização de empresas (Andersson, 2011; Harms \& Schiele, 2012; Sarasvathy, Kumar, York \& Bhagavatula, 2014; Galkina \& Chetty, 2015; Schweizer, 2015), é possível inferir que muitas das decisões acerca do empreendimento são frutos de heurísticas cognitivas (esquemas mentais) e abordagens lógicas (Sarasvathy, 2001a), com as quais os empreendedores exploram contingências, tomam decisões sob condições de incerteza e estabelecem interações (network) que são viabilizadas dentro e fora da organização (Gabrielsson \& Gabrielsson, 2013).

Os estudos evoluíram para uma concepção de que ambos os processos - Causation e Effectuation - não são excludentes entre si, podendo acontecer, alternativamente ou simultaneamente e com variações condicionadas ao contexto ou a escolhas dos próprios empreendedores (Harms \& Schiele, 2012). Em situações em que existam contingências que precisam ser superadas, mercados criados e redes de contato que viabilizem as alianças estratégicas (Gabrielsson \& Gabrielsson, 2013), em situações em que a percepção dos problemas exija o uso de lógicas simultâneas (Ciszewska-Mlinaric, Obloj \& Wasowska, 2016), em conjunturas que necessitem a sinergia (Smolka, Verheul, Burmeister-Lamp \& Heugens, 2016) e até mesmo em empresas com tamanhos diferentes, estabelecidas ou novatas ou com finalidades diferentes (Blekman, 2011), ou ainda, conforme Faiez \& Younes (2012, p.1413) reiteram, o "Effectuation é uma lógica de experiência empreendedora que tanto novatos como empreendedores experientes podem usar na fase altamente imprevisível no começo de um empreendimento (startup) para reduzir os custos de falhas para o empreendedor".

Estes estudos, sob certos aspectos, testificam a percepção de que as preferências dos empreendedores fazem com que uma abordagem (Causation ou Effectuation) não possa ser simplesmente considerada melhor ou pior que a outra, mas que a propensão está condicionada a fatores específicos, seja pelo contexto decisório ou mesmo por conjunturas do próprio empreendimento, mas qualquer escolha estará subordinada ao papel das heurísticas do empreendedor e as suas percepções acerca de cada alternativa (Schweizer, 2015).

\section{Método}

O presente estudo foi conduzido sob o enfoque qualitativo e teórico, visto a necessidade de entender com profundidade os temas. Deste modo, avaliamos as definições conceituais sobre o tema, as ações empreendedoras associadas às heurísticas dos empreendedores e de que forma as mesmas podem ser entendidas, sob enfoque teórico, como indicações de escolhas que irão determinar uma opção de uso de Causation, Effectuation ou uma combinação de ambas. Por conseguinte, os processos comportamentais envolvidos nestas ações oferecem a possibilidade de responder à questão fundamental que norteou este trabalho: Quais são as ações dos empreendedores que se caracterizam como comportamentos do tipo Causation e Effectuation? Para a definição dos artigos e ou outras bibliografias que fundamentaram estes temas, adotouse o seguinte processo: 1. Estabeleceu-se uma procura dos termos Causation e Effectuation e

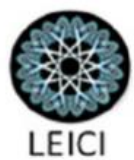


suas variações de busca ampliada na base de dados (Web of Science) com o propósito de selecionar intencionalmente 36 artigos entre 152 com maior número de citações entre os anos de 1999 a 2017. Provavelmente estes representam expertises teóricas dos temas e suas publicações teriam um alto fator de impacto. 2. Foram selecionados 36 artigos acerca do tema Causation e Effectuation, citados neste trabalho, onde se ressaltaram as seguintes particularidades: conceitos associados, contextos dos estudos, método de pesquisa adotado e o perfil metodológico, que - em conformidade com Machado-da-Silva, Cunha \& Amboni, 1990 - seriam os estudos do tipo empíricos, teóricos ou teórico-empíricos. 3. Também procuramos evidenciar os aspectos congruentes com o artigo seminal (Sarasvathy, 2001a) e os desafios de estudos futuros ou limitações apontadas pelos autores, podendo indicar caminhos que venham a integrar a teoria do Effectuation com outros conceitos teóricos, modelos e teorias relacionadas ao empreendedorismo, com vistas a colaborar para a compreensão do Effectuation, bem como para o campo mais amplo do empreendedorismo.

Com relação ao perfil metodológico, estes configuram "degraus" de profundidade, conforme, Machado-da-Silva, Cunha \& Amboni (1990) na Figura 2:

\begin{tabular}{|c|l|}
\hline Empíricos & $\begin{array}{l}\text { Definidos como aqueles focados em observação e análise dos dados (não } \\
\text { existe um quadro referencial específico que venha a explicar uma realidade). }\end{array}$ \\
\hline Teórico-empíricos & $\begin{array}{l}\text { São aqueles constituídos de um referencial teórico e, posteriormente, através, } \\
\text { da coleta de dados em campo, almejam confirmar ou mesmo refutar (total ou } \\
\text { parcialmente). }\end{array}$ \\
\hline Teóricos & $\begin{array}{l}\text { São aquelas pesquisas limitadas aos conceitos, proposições, construção de } \\
\text { modelos ou identificação de variáveis, não envolvendo exame empírico. }\end{array}$ \\
\hline
\end{tabular}

\section{Figura 2 - Perfil Metodológico.}

Fonte: Machado-da-Silva, Cunha \& Amboni. (1990).

Referente aos artigos de perfil metodológico teórico - empíricos, os mesmos ainda podem ser sub classificados em: Qualitativos, quantitativos ou mistos. (Creswell, 2009).

\section{Discussão}

Foram analisados 36 artigos, dentre os 152 mais citados nas bases de dados da Web of Science entre os anos de 1999 a 2017 sendo que 32 artigos $(88,89 \%)$ tem perfil metodológico teórico-empírico e $4(11,11 \%)$ são apenas teóricos, indicando uma teoria de base que têm subsidiado satisfatoriamente as pesquisas acerca dos comportamentos do tipo Causation e Effectuation. Destes, 14 (41,18\%) são qualitativos, 19 (52,78\%) quantitativos e $3(8,33 \%)$ são mistos (qualitativos e quantitativos) evidenciando que enquanto a quantidade dos estudos qualitativos e quantitativos tem sido razoavelmente equilibrados ainda são incipientes os mistos. Esta análise procurou examinar os enfoques destes trabalhos relacionados com as ações empreendedoras que se caracterizam como comportamentos do tipo Causation e Effectuation, nos contextos dos mais diversos e mapeou as pesquisas desenvolvidas no sentido de fluxo de pesquisas e suas particularidades: conceitos associados, contextos dos estudos, método de pesquisa adotado, perfil metodológico, os aspectos congruentes com o artigo seminal (Sarasvathy, 2001a) e os desafios de estudos futuros ou limitações apontadas pelos autores.

A Figura 3, a seguir, dispõe estes artigos em ordenamento alfabético: 


\begin{tabular}{|c|c|c|c|c|c|c|}
\hline Artigo & Conceitos associados & $\begin{array}{c}\text { Contextos dos } \\
\text { estudos }\end{array}$ & $\begin{array}{l}\text { Método } \\
\text { adotado }\end{array}$ & $\begin{array}{c}\text { Perfil } \\
\text { metodológico }\end{array}$ & $\begin{array}{l}\text { Aspectos congruentes } \\
\text { com Sarasvathy, 2001a }\end{array}$ & Desafios e limitações \\
\hline $\begin{array}{l}\text { Alsos \& Clausen } \\
\text { (2014). }\end{array}$ & $\begin{array}{l}\text { Expertise } \\
\text { empreendedora; } \\
\text { Ambidexteridade }\end{array}$ & $\begin{array}{l}\text { Startups de } \\
\text { empresas de } \\
\text { turismo. }\end{array}$ & Quantitativo & $\begin{array}{l}\text { Teórico- } \\
\text { empírico }\end{array}$ & $\begin{array}{l}\text { Causation e } \\
\text { Effectuation não são } \\
\text { excludentes. }\end{array}$ & $\begin{array}{l}\text { Confirmar se os resultados são restritos ao } \\
\text { ambiente empreendedor }\end{array}$ \\
\hline $\begin{array}{l}\text { Andersson } \\
(2011)\end{array}$ & $\begin{array}{l}\text { Conhecimento } \\
\text { empreendedor; } \\
\text { Internacionalização. } \\
\text { Networks }\end{array}$ & $\begin{array}{l}\text { Startup Born } \\
\text { globals }\end{array}$ & Qualitativo & $\begin{array}{l}\text { Teórico- } \\
\text { empírico }\end{array}$ & Alianças estratégicas & $\begin{array}{l}\text { Estudar não apenas as competências; aprofundar } \\
\text { para identificar comportamento }\end{array}$ \\
\hline $\begin{array}{l}\text { Appelhoff Mauer, } \\
\text { Collewaert\& } \\
\text { Brettel (2015). }\end{array}$ & $\begin{array}{l}\text { Conflito entre } \\
\text { empreendedora e } \\
\text { investidor }\end{array}$ & $\begin{array}{l}\text { Empresas alemãs } \\
\text { com investimento } \\
\text { anjo. }\end{array}$ & Quantitativo & $\begin{array}{l}\text { Teórico- } \\
\text { empírico }\end{array}$ & $\begin{array}{l}\text { Princípios do } \\
\text { Effectuation: Perda } \\
\text { acessível }\end{array}$ & Estudar as causas dos conflitos \\
\hline $\begin{array}{l}\text { Blauth, Mauer \& } \\
\text { Brettel (2014). }\end{array}$ & $\begin{array}{l}\text { Processos criativos no } \\
\text { desenvolvimento de } \\
\text { novos produtos;Decisão } \\
\text { empreendedora; }\end{array}$ & $\begin{array}{l}\text { Empresas alemãs } \\
\text { de P\&D }\end{array}$ & Quantitativo & $\begin{array}{l}\text { Teórico- } \\
\text { empírico }\end{array}$ & $\begin{array}{l}\text { Controle de um futuro } \\
\text { imprevisível; Tomada } \\
\text { de decisão sob incerteza } \\
\text { (Effectuation) }\end{array}$ & $\begin{array}{l}\text { Recomendou-se uma pesquisa longitudinal; } \\
\text { Recomenda-se adotar outro meio de pesquisa que } \\
\text { diversifique a faixa etária dos respondentes. }\end{array}$ \\
\hline Blekman (2011). & $\begin{array}{l}\text { Empreendedorismo } \\
\text { corporativo }\end{array}$ & Empresas & Qualitativo & Teórico & $\begin{array}{l}\text { Princípios do } \\
\text { Effectuation }\end{array}$ & $\begin{array}{l}\text { Recomenda-se a aplicação das idéias em múltiplos } \\
\text { contextos empresariais }\end{array}$ \\
\hline $\begin{array}{l}\text { Brettel, Mauer, } \\
\text { Engelen \& } \\
\text { Küpper (2012). }\end{array}$ & $\begin{array}{l}\text { Processos de P \& D e o } \\
\text { desempenho do projeto }\end{array}$ & $\begin{array}{l}\text { Projetos de P\&D } \\
\text { em empresas }\end{array}$ & $\begin{array}{l}\text { Qualitativo } \\
+ \\
\text { quantitativo. }\end{array}$ & $\begin{array}{l}\text { Teórico- } \\
\text { empírico }\end{array}$ & $\begin{array}{l}3 \text { Princípios do } \\
\text { Effectuation são } \\
\text { predominantes }\end{array}$ & $\begin{array}{l}\text { Recomenda-se pesquisas longitudinais; Incorporar } \\
\text { outras medidas de desempenho do conjunto de } \\
\text { projetos de P\&D. }\end{array}$ \\
\hline $\begin{array}{l}\text { Chandler, } \\
\text { DeTienne, } \\
\text { McKelvie \& } \\
\text { Mumford (2011). }\end{array}$ & $\begin{array}{l}\text { Causation e } \\
\text { Effectuation para a } \\
\text { criação de novos } \\
\text { negócios }\end{array}$ & $\begin{array}{l}\text { Curso de MBA e } \\
\text { empreendedores. }\end{array}$ & $\begin{array}{l}\text { Qualitativo } \\
+ \\
\text { quantitativo. }\end{array}$ & $\begin{array}{l}\text { Teórico- } \\
\text { empírico }\end{array}$ & $\begin{array}{l}\text { Princípios do } \\
\text { Effectuation; } \\
\text { Effectuation } \\
\text { predominante na } \\
\text { incerteza. }\end{array}$ & $\begin{array}{l}\text { Autores recomendaram replicação da pesquisa em } \\
\text { outros contextos; negócios iniciais; Pesquisar se } \\
\text { afeta a orientação empreendedora. }\end{array}$ \\
\hline $\begin{array}{l}\text { Ciszewska- } \\
\text { Mlinaric, Obloj \& } \\
\text { Wasowska (2016) }\end{array}$ & $\begin{array}{l}\text { Lógica de tomada de } \\
\text { decisão em empresas } \\
\text { em processo de } \\
\text { internacionalização. }\end{array}$ & $\begin{array}{l}\text { Empresa } \\
\text { polonesa: caso } \\
\text { único. }\end{array}$ & Qualitativo. & $\begin{array}{l}\text { Teórico- } \\
\text { empírico. }\end{array}$ & $\begin{array}{l}\text { Causation e } \\
\text { Effectuation não são } \\
\text { excludentes. }\end{array}$ & $\begin{array}{l}\text { Para orientar e manter um empreendedor } \\
\text { Effectual, recomenda-se que novos desafios sejam } \\
\text { explorados, mas com objetivos claros. }\end{array}$ \\
\hline
\end{tabular}

Organizadores:

- fav eaesp 


\begin{tabular}{|c|c|c|c|c|c|c|}
\hline $\begin{array}{l}\text { da Costa \& } \\
\text { Brettel (2011). }\end{array}$ & $\begin{array}{l}\text { Comportamento } \\
\text { inovador; pro atividade } \\
\text { e tenacidade; Efeito dos } \\
\text { traços de personalidade }\end{array}$ & $\begin{array}{l}\text { Empresa } \\
\text { multinacional de } \\
\text { grande porte. }\end{array}$ & Quantitativo & $\begin{array}{l}\text { Teórico- } \\
\text { empírico. }\end{array}$ & $\begin{array}{l}\text { Causation e } \\
\text { Effectuation não são } \\
\text { excludentes. }\end{array}$ & $\begin{array}{l}\text { Para estudos futuros sugerem pesquisar a } \\
\text { influência de equipes e interações em } \\
\text { os times sobre o comportamento do grupo. }\end{array}$ \\
\hline $\begin{array}{l}\text { Deligianni } \\
\text { Voudouris \& } \\
\text { Lioukas (2015). }\end{array}$ & $\begin{array}{l}\text { Performance de novos } \\
\text { negócios; Effectuation } \\
\text { modera a diversificação. }\end{array}$ & $\begin{array}{l}\text { Novos negócios } \\
\text { gregos. }\end{array}$ & Quantitativo & $\begin{array}{l}\text { Teórico- } \\
\text { empírico. }\end{array}$ & $\begin{array}{l}\text { Eexperimentação, } \\
\text { flexibilidade e pré- } \\
\text { compromissos. }\end{array}$ & $\begin{array}{l}\text { A perda acessível deve ser investigada, em que as } \\
\text { diferenças nas percepções de risco podem ser mais } \\
\text { facilmente entendidas }\end{array}$ \\
\hline $\begin{array}{l}\text { DeTienne \& } \\
\text { Chandler (2010). }\end{array}$ & $\begin{array}{l}\text { O Impacto da } \\
\text { Motivação e das } \\
\text { Abordagens nas } \\
\text { Estratégias de Saída. }\end{array}$ & $\begin{array}{l}\text { Duas Empresas } \\
\text { industriais. }\end{array}$ & Quantitativo & $\begin{array}{l}\text { Teórico- } \\
\text { empírico. }\end{array}$ & $\begin{array}{l}\text { Causation e } \\
\text { Effectuation não são } \\
\text { excludentes. }\end{array}$ & $\begin{array}{l}\text { Limitação do trabalho é ter sido feita com método } \\
\text { único em poucas indústrias, autores recomendam } \\
\text { diversificar a amostra e o método. }\end{array}$ \\
\hline $\begin{array}{l}\text { Dew, Read, } \\
\text { Sarasvathy \& } \\
\text { Wiltbank (2008). }\end{array}$ & $\begin{array}{l}\text { Teoria comportamental } \\
\text { com a Expertise } \\
\text { empreendedora. }\end{array}$ & Ensaio teórico. & Qualitativo. & Teórico. & $\begin{array}{l}\text { Princípios do } \\
\text { Effectuation }\end{array}$ & $\begin{array}{l}\text { Processos futuros deveriam buscar entender como } \\
\text { as decisões são tomadas pelas pessoas com ou sem } \\
\text { ajuda da organização; }\end{array}$ \\
\hline $\begin{array}{l}\text { Dew, Read, } \\
\text { Sarasvathy\& } \\
\text { Wiltbank (2009). }\end{array}$ & $\begin{array}{l}\text { Diferenças de processos } \\
\text { decisórios Causation e } \\
\text { Effectuation entre } \\
\text { experts e novatos. }\end{array}$ & $\begin{array}{l}27 \\
\text { empreendedores } \\
\text { experts com } 37 \\
\text { alunos MBA. } \\
\end{array}$ & Quantitativo & $\begin{array}{l}\text { Teórico- } \\
\text { empírico. }\end{array}$ & $\begin{array}{l}\text { Princípios do } \\
\text { Effectuation: perda } \\
\text { acessível. }\end{array}$ & $\begin{array}{l}\text { Autores sugerem mais pesquisas cognitivas que } \\
\text { venham a confirmar ou refutar os resultados que } \\
\text { apontaram que experts são essencialmente } \\
\text { Effectuators. }\end{array}$ \\
\hline $\begin{array}{l}\text { Engel, Dimitrova, } \\
\text { Khapova \& } \\
\text { Elfring (2014). }\end{array}$ & $\begin{array}{l}\text { Auto-eficácia } \\
\text { empreendedora entre } \\
\text { experts e novatos. }\end{array}$ & $\begin{array}{l}93 \text { estudantes de } \\
\text { negócios em uma } \\
\text { universidade } \\
\text { holandesa. }\end{array}$ & Quantitativo & $\begin{array}{l}\text { Teórico- } \\
\text { empírico. }\end{array}$ & $\begin{array}{l}\text { Princípios do } \\
\text { Effectuation. }\end{array}$ & $\begin{array}{l}\text { Foi entendido pelos autores a contribuição baseada } \\
\text { em estudos de empreendedores inexperientes } \\
\text { como um avanço teórico e empírico. }\end{array}$ \\
\hline $\begin{array}{l}\text { Evers \& } \\
\text { O'Gorman (2011) }\end{array}$ & $\begin{array}{l}\text { Effectuation e a } \\
\text { improvisação no } \\
\text { contexto do processo de } \\
\text { internacionalização. }\end{array}$ & $\begin{array}{l}3 \text { empresas } \\
\text { irlandesas que } \\
\text { processam } \\
\text { mariscos. }\end{array}$ & Qualitativo. & $\begin{array}{l}\text { Teórico- } \\
\text { empírico. }\end{array}$ & $\begin{array}{l}\text { Princípios do } \\
\text { Effectuation. }\end{array}$ & $\begin{array}{l}\text { Sugere-se expandir pesquisas em um outro } \\
\text { contexto geográfico dado; suspeita-se que quanto } \\
\text { mais internacional o contexto, em indivíduos, } \\
\text { empresas e instituições, mais provável é que o } \\
\text { conhecimento idiossincrático necessário para a } \\
\text { internacionalização seja desenvolvido. }\end{array}$ \\
\hline $\begin{array}{l}\text { Faiez \& Younes } \\
\text { (2012). }\end{array}$ & $\begin{array}{l}\text { Uma abordagem } \\
\text { cognitiva para analisar a } \\
\text { influência da rede } \\
\text { effectual. sobre as ações } \\
\text { empreendedoras. }\end{array}$ & $\begin{array}{l}7 \text { empreendedores } \\
\text { Tunisianos } \\
\text { (Tunísia). }\end{array}$ & Qualitativo. & $\begin{array}{l}\text { Teórico- } \\
\text { empírico. }\end{array}$ & $\begin{array}{l}\text { Princípios do } \\
\text { Effectuation: } \\
\text { principalmente o uso da } \\
\text { rede social. (Alianças } \\
\text { estratégicas). }\end{array}$ & $\begin{array}{l}\text { Direções futuras para a análise cognitiva de } \\
\text { effectuation e network effectual. }\end{array}$ \\
\hline
\end{tabular}




\section{XEGEPE

\begin{tabular}{|c|c|c|c|c|c|c|}
\hline Artigo & Conceitos associados & $\begin{array}{c}\text { Contextos dos } \\
\text { estudos }\end{array}$ & $\begin{array}{r}\text { Método } \\
\text { adotado }\end{array}$ & $\begin{array}{c}\text { Perfil } \\
\text { metodológico }\end{array}$ & $\begin{array}{l}\text { Aspectos congruentes } \\
\text { com Sarasvathy, 2001a }\end{array}$ & Desafios e limitações \\
\hline Fisher (2012). & $\begin{array}{l}\text { Dimensões } \\
\text { comportamentais } \\
\text { comuns de effectuation } \\
\text { e bricolagem } \\
\text { empreendedora. }\end{array}$ & $\begin{array}{l}\text { Teórico, baseado } \\
\text { em informações } \\
\text { de } 6 \text { empresas de } \\
\text { internet. }\end{array}$ & Qualitativo. & $\begin{array}{l}\text { Teórico - } \\
\text { empírico. }\end{array}$ & $\begin{array}{l}\text { Princípios do } \\
\text { Effectuation; Corrobora } \\
\text { a relevância do } \\
\text { comportamento e das } \\
\text { heurísticas do } \\
\text { empreendedor. }\end{array}$ & $\begin{array}{l}\text { Pesquisas futuras poderiam identificar e } \\
\text { desenvolver novas proposições focadas nas } \\
\text { relações entre recursos, oportunidades } \\
\text { empreendedoras, ação, soluções, comunidades, } \\
\text { restrições de recursos e criatividade que podem } \\
\text { ainda mais enriquecer a pesquisa empreendedora. }\end{array}$ \\
\hline $\begin{array}{l}\text { Gabrielsson \& } \\
\text { Politis (2011). }\end{array}$ & $\begin{array}{l}\text { Motivação de carreira e } \\
\text { tomada de decisões } \\
\text { empreendedoras em } \\
\text { novos } \\
\text { empreendimentos. }\end{array}$ & $\begin{array}{l}291 \text { indivíduos } \\
\text { em empresas } \\
\text { suecas. }\end{array}$ & Quantitativo & $\begin{array}{l}\text { Teórico - } \\
\text { empírico. }\end{array}$ & $\begin{array}{l}\text { Causation e } \\
\text { Effectuation não são } \\
\text { excludentes. }\end{array}$ & $\begin{array}{l}\text { Os autores sugerem estudos adicionais; pode ser } \\
\text { interessante estudar de perto o papel da } \\
\text { experiência educacional e de trabalho na } \\
\text { determinação de variações nas motivações de } \\
\text { carreira, tanto quando os indivíduos entram, } \\
\text { quanto persistem em auto emprego. }\end{array}$ \\
\hline $\begin{array}{l}\text { Galkina \& Chetty } \\
\text { (2015). }\end{array}$ & $\begin{array}{l}\text { Processo de } \\
\text { internacionalização de } \\
\text { PME's. }\end{array}$ & $\begin{array}{l}7 \text { empresas } \\
\text { Finlandesas em } \\
\text { processo de } \\
\text { internacionalizaçã } \\
\text { o. }\end{array}$ & Qualitativo. & $\begin{array}{l}\text { Teórico - } \\
\text { empírico. }\end{array}$ & $\begin{array}{l}\text { Causation e } \\
\text { Effectuation não são } \\
\text { excludentes.: } \\
\text { principalmente o uso da } \\
\text { rede social (Alianças } \\
\text { estratégicas) }\end{array}$ & $\begin{array}{l}\text { As autoras ressaltam a evolução dos estudos para } \\
\text { entendimento dos aspectos complexos das redes } \\
\text { em contextos de internacionalização (construçães } \\
\text { de compromissos, oportunidades, confiança, } \\
\text { incerteza e coordenação) }\end{array}$ \\
\hline $\begin{array}{l}\text { Harms \& Schiele } \\
\text { (2012). }\end{array}$ & $\begin{array}{l}\text { Seleção de modo de } \\
\text { entrada em mercados } \\
\text { internacionais; } \\
\text { Empreendedorismo } \\
\text { corporativo. }\end{array}$ & $\begin{array}{l}65 \text { PME's em } \\
\text { rápido } \\
\text { crescimento (os } \\
\text { finalistas do } \\
\text { concurso alemão } \\
\text { "Empreendedor } \\
\text { do Ano" } 2010 . \\
\end{array}$ & Quantitativo & $\begin{array}{l}\text { Teórico - } \\
\text { empírico. }\end{array}$ & $\begin{array}{l}\text { Causation e } \\
\text { Effectuation não são } \\
\text { excludentes. Podem ser } \\
\text { simultâneos. }\end{array}$ & $\begin{array}{l}\text { Os autores sugerem que os conceitos Causation e } \\
\text { Effectuation não devem ser tomados como opostos } \\
\text { e poderiam ser utilizados ao mesmo tempo. } \\
\text { Propõem que se analisem em trabalhos futuros } \\
\text { diferentes antecedentes e consequentes de } \\
\text { Causation e Effectuation no processo } \\
\text { Internacionalização, por exemplo, sobre } \\
\text { precocidade, velocidade e escopo. }\end{array}$ \\
\hline $\begin{array}{l}\text { Johansson \& } \\
\text { McKelvie (2012). }\end{array}$ & $\begin{array}{l}\text { Capital humano em } \\
\text { contexto corporativo. }\end{array}$ & $\begin{array}{l}246 \text { indivíduos de } \\
\text { empresas suecas } \\
\text { de capital aberto. }\end{array}$ & Quantitativo & $\begin{array}{l}\text { Teórico - } \\
\text { empírico. }\end{array}$ & $\begin{array}{l}\text { Causation e } \\
\text { Effectuation não são } \\
\text { excludentes. Presença } \\
\text { de ambidexteridade. }\end{array}$ & $\begin{array}{l}\text { Os autores apontam caminhos de pesquisa } \\
\text { complementares acerca dos determinantes da } \\
\text { escolha das lógicas de tomada de decisão que } \\
\text { podem ser empregadas para buscar melhores } \\
\text { oportunidades em condições de incerteza. }\end{array}$ \\
\hline
\end{tabular}




\section{ExGePE}

\begin{tabular}{|c|c|c|c|c|c|c|}
\hline $\begin{array}{l}\text { Kalinic, } \\
\text { Sarasvathy\& } \\
\text { Forza (2014). }\end{array}$ & $\begin{array}{l}\text { Tomada de decisão } \\
\text { effectual no processo de } \\
\text { internacionalização. }\end{array}$ & $\begin{array}{l}5 \text { empresas } \\
\text { manufatureiras } \\
\text { italianas. }\end{array}$ & Qualitativo. & $\begin{array}{l}\text { Teórico - } \\
\text { empírico. }\end{array}$ & $\begin{array}{l}\text { Princípios do } \\
\text { Effectuation.Com } \\
\text { evidências fortes do uso } \\
\text { do princípio de } \\
\text { superação de } \\
\text { contingências. }\end{array}$ & $\begin{array}{l}\text { O foco deve ser o conceito de perda acessível e } \\
\text { como lidar com ele de um ponto de vista prático } \\
\text { (por exemplo, como calcular). Finalmente, os } \\
\text { empreendedores devem ser capazes de identificar } \\
\text { condições quando não é possível basear suas } \\
\text { decisões na lógica da mitigação de custos e retorno } \\
\text { de investimento ajustado ao risco. }\end{array}$ \\
\hline $\begin{array}{l}\text { Laine \& Galkina } \\
\text { (2017). }\end{array}$ & $\begin{array}{l}\text { Incerteza Institucional } \\
\text { (Teoria Institucional) } \\
\text { Internacionalização de } \\
\text { PME's. }\end{array}$ & $\begin{array}{l}4 \text { empresas de } \\
\text { setores diferentes } \\
\text { situadas na } \\
\text { Rússia. }\end{array}$ & Qualitativo. & $\begin{array}{l}\text { Teórico - } \\
\text { empírico. }\end{array}$ & $\begin{array}{l}\text { Princípios do } \\
\text { Effectuation. }\end{array}$ & $\begin{array}{l}\text { Os autores apontam que o estudo fornece algumas } \\
\text { evidências de que a incerteza estrutural impulsiona } \\
\text { o Effectuation, enquanto a incerteza dos } \\
\text { parâmetros pode ser abordada com Causation, } \\
\text { pode desencadear uma direção interessante para } \\
\text { novas pesquisas sobre tomada de decisão sob } \\
\text { vários tipos de incerteza. }\end{array}$ \\
\hline $\begin{array}{l}\text { Laskovaia, } \\
\text { Shirokova \& } \\
\text { Morris (2017). }\end{array}$ & $\begin{array}{l}\text { Cultura e tomada de } \\
\text { decisão empreendedora. }\end{array}$ & $\begin{array}{l}3411 \text { empresas de } \\
24 \text { países. }\end{array}$ & Quantitativo & $\begin{array}{l}\text { Teórico - } \\
\text { empírico. }\end{array}$ & $\begin{array}{l}\text { Princípios do } \\
\text { Effectuation; Causation } \\
\text { e Effectuation não são } \\
\text { excludentes e podem } \\
\text { ocorrer } \\
\text { simultaneamente. }\end{array}$ & $\begin{array}{l}\text { A pesquisa futura deve levar em conta a } \\
\text { possibilidade de mudanças nos padrões culturais } \\
\text { inerentes a locais particulares ou mudanças nas } \\
\text { proporções de diferentes dimensões culturais que } \\
\text { constituem normas culturais de ordem superior. } \\
\text { Recomendam-se pesquisas longitudinais que } \\
\text { avaliem também outros conjuntos de respondentes } \\
\text { para que se possa constatar o uso de lógicas } \\
\text { Causation e Effectuation por estes indivíduos. }\end{array}$ \\
\hline $\begin{array}{l}\text { Long, Xia \& Hu } \\
\text { (2017). }\end{array}$ & $\begin{array}{l}\text { Descoberta de } \\
\text { oportunidades; } \\
\text { Inovações. }\end{array}$ & $\begin{array}{l}\text { Empresas } \\
\text { chinesas. , } 8 \\
\text { cidades. }\end{array}$ & Quantitativo & $\begin{array}{l}\text { Teórico - } \\
\text { empírico. }\end{array}$ & $\begin{array}{l}\text { Princípios do } \\
\text { Effectuation a tomada } \\
\text { de decisão. }\end{array}$ & $\begin{array}{l}\text { Os autores recomendam maiores estudos que } \\
\text { avaliem o efeito das interações nos estudos de } \\
\text { Effectuation. }\end{array}$ \\
\hline $\begin{array}{l}\text { Read, Dew, } \\
\text { Sarasvathy, Song } \\
\text { \& Wiltbank } \\
(2009) .\end{array}$ & $\begin{array}{l}\text { Tomada de decisão em } \\
\text { Marketing. }\end{array}$ & $\begin{array}{l}27 \\
\text { empreendedores } \\
+37 \text { gerentes. }\end{array}$ & Quantitativo & $\begin{array}{l}\text { Teórico - } \\
\text { empírico. }\end{array}$ & $\begin{array}{l}\text { Princípios do } \\
\text { Effectuation como } \\
\text { logica preferencial, mas } \\
\text { dicotômico em relação } \\
\text { ao Causation. }\end{array}$ & $\begin{array}{l}\text { Pesquisas adicionais podem investigar estratégias } \\
\text { e metas efetivas para a cocriação. Por exemplo, } \\
\text { apesar de muita literatura ter examinado parceiros } \\
\text { de financiamentos, um fornecedor crítico pode ser } \\
\text { um parceiro de cocriação mais importante para um } \\
\text { novo empreendimento que, normalmente, não } \\
\text { possui recursos e conhecimentos. Outras pesquisas } \\
\text { também podem examinar as condições e as }\end{array}$ \\
\hline
\end{tabular}

UNICAMP 


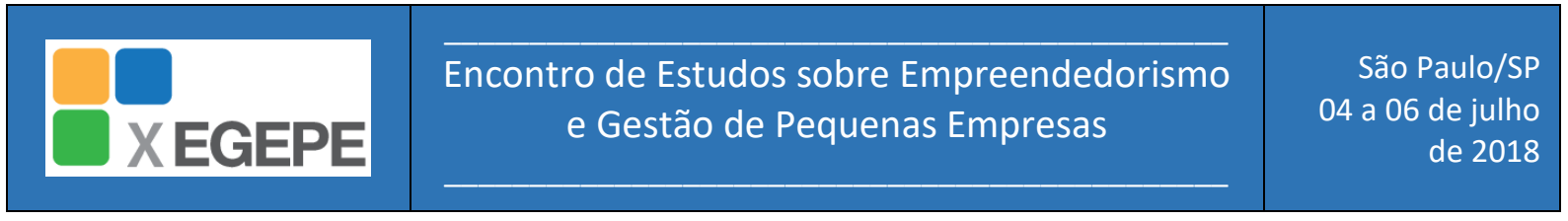

\begin{tabular}{|c|c|c|c|c|c|c|}
\hline & & & & & & $\begin{array}{l}\text { abordagens nas quais a pesquisa de mercado } \\
\text { melhora o sucesso do novo empreendimento. }\end{array}$ \\
\hline $\begin{array}{l}\text { Roach, Ryman \& } \\
\text { Makani (2016). }\end{array}$ & $\begin{array}{l}\text { Princípios do } \\
\text { Effectuation no } \\
\text { contexto de inovação. }\end{array}$ & $\begin{array}{l}\text { Gerentes seniores } \\
\text { de } 169 \text { PME's } \\
\text { americanas. }\end{array}$ & Quantitativo & $\begin{array}{l}\text { Teórico - } \\
\text { empírico. }\end{array}$ & $\begin{array}{l}\text { Princípios do } \\
\text { Effectuation. }\end{array}$ & $\begin{array}{l}\text { Os autores propõem pesquisas futuras com base } \\
\text { em medidas de inovação usadas no estudo. }\end{array}$ \\
\hline $\begin{array}{l}\text { Sarasvathy } \\
\text { (2001b). }\end{array}$ & $\begin{array}{l}\text { Tomada de decisão; } \\
\text { Effectuation. }\end{array}$ & $\begin{array}{l}\text { empreendedores } \\
\text { especialistas de } \\
\text { grandes empresas } \\
\text { americanas. (27) }\end{array}$ & Quantitativo & $\begin{array}{l}\text { Teórico- } \\
\text { empírico. }\end{array}$ & $\begin{array}{l}\text { Causation e } \\
\text { Effectuation são } \\
\text { inversos (dicotômicos). }\end{array}$ & $\begin{array}{l}\text { A autora sugere a adoção do Effectuation como } \\
\text { modelo de decisão empreendedora. }\end{array}$ \\
\hline $\begin{array}{l}\text { Sarasvathy } \\
(2008) \text {. }\end{array}$ & $\begin{array}{l}\text { Tomada de decisão; } \\
\text { Effectuation. }\end{array}$ & Teórico. & Qualitativo. & Teórico. & $\begin{array}{l}\text { Causation e } \\
\text { Effectuation são } \\
\text { inversos (dicotômicos). }\end{array}$ & $\begin{array}{l}\text { A autora sugere a adoção do Effectuation como } \\
\text { modelo de decisão empreendedora. }\end{array}$ \\
\hline $\begin{array}{l}\text { Sarasvathy\& Dew } \\
\text { (2005). }\end{array}$ & $\begin{array}{l}\text { Novos mercados; Rede } \\
\text { social; Stakeholders. }\end{array}$ & Teórico. & Qualitativo. & Teórico. & $\begin{array}{l}\text { Effectuation como } \\
\text { lógica preferencial de } \\
\text { tomada de decisão. }\end{array}$ & $\begin{array}{l}\text { Os autores sugerem a adoção do Effectuation } \\
\text { como modelo de decisão empreendedora, em } \\
\text { situações de incerteza. }\end{array}$ \\
\hline $\begin{array}{l}\text { Sarasvathy, } \\
\text { Kuma, York \& } \\
\text { Bhagavatul } \\
\text { (2014). }\end{array}$ & $\begin{array}{l}\text { Empreendedorismo } \\
\text { internacional. }\end{array}$ & $\begin{array}{l}\text { Estudo de caso } \\
\text { único empresa } \\
\text { indiana. }\end{array}$ & Qualitativo. & $\begin{array}{l}\text { Teórico- } \\
\text { empírico. }\end{array}$ & $\begin{array}{l}\text { Princípios do } \\
\text { Effectuation. }\end{array}$ & $\begin{array}{l}\text { Os autores apontam quatro possibilidades como } \\
\text { forma de iniciar uma conversa emocionante: } 1 \text {. Em } \\
\text { termos de generalização: para empreendimentos } \\
\text { sociais e formas organizacionais inovadoras; } 2 \text {. } \\
\text { Em termos de método: para análises } \\
\text { contrafactuais; 3. Em termos de unidade de } \\
\text { análise: em direção ao intersubjetivo; } 4 \text {. Em } \\
\text { termos de escopo: para empreendimentos } \\
\text { transnacionais e pós-nacionais. }\end{array}$ \\
\hline Schweizer (2015). & $\begin{array}{l}\text { Tomada de decisão } \\
\text { Internacionalização; } \\
\text { Modelos mentais. }\end{array}$ & $\begin{array}{l}\text { Estudo de caso } \\
\text { empresa sueca. }\end{array}$ & Qualitativo. & $\begin{array}{l}\text { Teórico- } \\
\text { empírico. }\end{array}$ & $\begin{array}{l}\text { Presença de } \\
\text { ambidexteridade. }\end{array}$ & $\begin{array}{l}\text { Estudar a tomada de decisão dentro das lógicas } \\
\text { Causation e Effectuation em outros tipos de } \\
\text { organizações que queiram internacionalizar. }\end{array}$ \\
\hline $\begin{array}{l}\text { Smolka, Verheul, } \\
\text { Burmeister-Lamp } \\
\text { \& Heugens } \\
(2016) .\end{array}$ & $\begin{array}{l}\text { Tomada de decisão } \\
\text { empreendedora; } \\
\text { Sinergia entre } \\
\text { Causation e } \\
\text { Effectuation. }\end{array}$ & $\begin{array}{l}1453 \\
\text { empreendedores } \\
\text { de } 25 \text { países. }\end{array}$ & Quantitativo & $\begin{array}{l}\text { Teórico- } \\
\text { empírico. }\end{array}$ & $\begin{array}{l}\text { Causation e } \\
\text { Effectuation não são } \\
\text { excludentes. Presença } \\
\text { de ambidexteridade. }\end{array}$ & $\begin{array}{l}\text { Autores recomendam maiores estudos sobre a } \\
\text { capacidade de um empreendedor de alavancar } \\
\text { contingências ou construir habilidades de } \\
\text { parcerias; Estudar antecedentes e consequentes do } \\
\text { Effectuation. }\end{array}$ \\
\hline
\end{tabular}

-rgv eaesp 


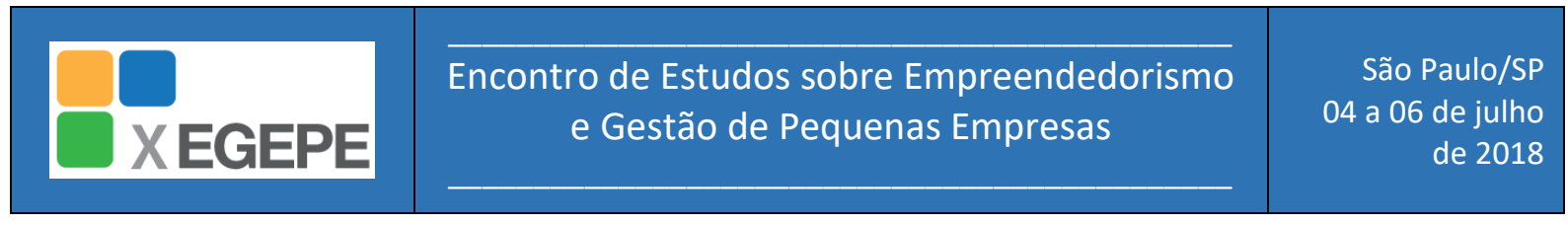

\begin{tabular}{|c|c|c|c|c|c|c|}
\hline $\begin{array}{l}\text { Welter, Mauer \& } \\
\text { Wuebker (2015). }\end{array}$ & $\begin{array}{l}\text { Effectuation e } \\
\text { bricolagem na Criação } \\
\text { de oportunidades, }\end{array}$ & $\begin{array}{l}\text { Dados do estudo } \\
\text { GUESSS } \\
\text { coletados entre } \\
\text { março e maio de } \\
2011 \text { de } 489 \\
\text { universidades em } \\
26 \text { países. } 1.453 \\
\text { indivíduos. } \\
\end{array}$ & Quantitativo & $\begin{array}{l}\text { Teórico- } \\
\text { empírico. }\end{array}$ & $\begin{array}{l}\text { Princípios do } \\
\text { Effectuation. }\end{array}$ & $\begin{array}{l}\text { Os autores evidenciam a necessidade de maiores } \\
\text { pesquisas que utilizem os conceitos associados à } \\
\text { criatividade em empreendedorismo e gestão. } \\
\text { Também sugerem diferenciar de forma mais clara } \\
\text { os aspectos de Incerteza Knightiana e a } \\
\text { racionalidade limitada. }\end{array}$ \\
\hline $\begin{array}{l}\text { Werhahn, Mauer, } \\
\text { Flatten \& Brettel } \\
\text { (2015). }\end{array}$ & $\begin{array}{l}\text { Escala } \\
\text { multidimensional } \\
\text { Effectual; } \\
\text { Effectuation em nível } \\
\text { organizacional; } \\
\text { Orientação estratégica. }\end{array}$ & $\begin{array}{l}421 \text { empresas } \\
\text { alemãs. }\end{array}$ & $\begin{array}{l}\text { Qualitativo } \\
\mathrm{e} \\
\text { Quantitativo }\end{array}$ & $\begin{array}{l}\text { Teórico- } \\
\text { empírico. }\end{array}$ & $\begin{array}{l}\text { Princípios do } \\
\text { Effectuation. }\end{array}$ & $\begin{array}{l}\text { Os autores sugerem pesquisas futuras para } \\
\text { explorar os fatores que permitem a implementação } \\
\text { comportamental de orientação Effectual. }\end{array}$ \\
\hline $\begin{array}{l}\text { Wiltbank, Read, } \\
\text { Dew \& } \\
\text { Sarasvathy } \\
\text { (2009). }\end{array}$ & $\begin{array}{l}\text { Investimento anjo; } \\
\text { Novos negócios. }\end{array}$ & $\begin{array}{l}121 \text { investidores } \\
\text { anjo americanos. }\end{array}$ & Quantitativo & $\begin{array}{l}\text { Teórico- } \\
\text { empírico. }\end{array}$ & $\begin{array}{l}\text { Princípios do } \\
\text { Effectuation. }\end{array}$ & $\begin{array}{l}\text { O estudo atual evidenciou a possibilidade } \\
\text { intrigante de que os investidores anjo possam } \\
\text { limitar seus fracassos negativos através de uma } \\
\text { abordagem baseada em controle, um movimento } \\
\text { de redução de risco. Isso, segundo os autores } \\
\text { demandaria mais estudos futuros, ao estudar } \\
\text { diferentes performances com diferentes lógicas de } \\
\text { decisão sendo utilizadas. }\end{array}$ \\
\hline
\end{tabular}

Figura 3 - Análises dos fluxos e particularidades da pesquisa de Causation Effectuation

Fonte: $\mathrm{O}$ autor. 
Ao analisar os fluxos de pesquisas destes 36 artigos selecionados que estudam as abordagens Causation ou Effectuation e sua opção de utilização pelos empreendedores, percebe-se que muitas das pesquisas evidenciam que estas abordagens não podem ser entendidas como dicotômicas ou excludentes entre si DeTienne \& Chandler (2010) Gabrielsson \& Politis (2011), da Costa \& Brettel (2011), Harms \& Schiele (2012). Johansson \& McKelvie (2012), Alsos \& Clausen (2014), Galkina \& Chetty (2015), Schweizer (2015), CiszewskaMlinaric, Obloj \& Wasowska (2016), Smolka, Verheul, Burmeister-Lamp \& Heugens (2016) e Laskovaia, Shirokova \& Morris (2017). Assim, ao se referir a uma ou a outra, ambas podem ser percebidas como complementares, concomitantes, ou, ainda, sequenciais ou simultâneas.

Contrariamente, Sarasvathy (2001b), Sarasvathy (2008), Dew, Read, Sarasvathy \& Wiltbank (2008) e Read, Dew, Sarasvathy, Song \& Wiltbank (2009) entendem que ambas as abordagens são opostas e que as ações empreendedoras são baseadas nas heurísticas e na expertise dos empreendedores ajustados ao artigo seminal de Sarasvathy(2001a) que também tinha este viés. Desta maneira, os comportamentos empreendedores do tipo Effectuation estariam, nestas pesquisas, vinculados à expertise (preferencialmente em negócios anteriores), o que tornaria o empreendedor mais maleável para enfrentar os riscos, negligenciando o planejamento formal (empreendedores com comportamento do tipo Causation) e propenso a estabelecer parcerias para enfrentamento das contingências e para viabilização de novos negócios.

Outros artigos abordam o Effectuation como lógica preferencial para as ações empreendedoras e entendem que tanto os empreendedores experts como os novatos podem adotar esta abordagem de ação, caracterizada pela tolerância às perdas, a valorização das alianças estratégicas, a exploração de contingências e na imprevisibilidade do futuro. (Sarasvathy\& Dew, 2005, Wiltbank, Read, Dew \& Sarasvathy, 2009, Andersson, 2011, Blekman, 2011, Chandler, DeTienne, McKelvie \& Mumford, 2011, Evers \& O'Gorman (2011), Brettel, Mauer, Engelen \& Küpper (2012), Faiez \& Younes (2012), Fisher, 2012, Blauth, Mauer \& Brettel, 2014, Engel, Dimitrova, Khapova \& Elfring, 2014, Kalinic, Sarasvathy\& Forza, 2014, Sarasvathy, Kuma, York \& Bhagavatul, 2014, Appelhoff Mauer, Collewaert\& Brettel, 2015, Deligianni Voudouris \& Lioukas, 2015, Welter, Mauer \& Wuebker, 2015, Werhahn, Mauer, Flatten \& Brettel, 2015, Roach, Ryman \& Makani, 2016, Laine \& Galkina, 2017 e Long, $\mathrm{Xia} \& \mathrm{Hu}, 2017)$.

Deste modo, as ações dos empreendedores que se caracterizam como comportamentos do tipo Causation e Effectuation apesar de serem diferentes e dependentes do contexto, podem ser concebidas como complementares.

\section{Considerações Finais}

O empreendedorismo é um tema que vem demandando estudos e pesquisas em diferentes contextos, e as diferentes teorias existentes requerem profundidade e consolidação, abrindo áreas de estudo diversas. Algumas delas enveredam para cenários cujo empreendedor procura identificar formas para gerir suas organizações e, assim, assimilar maior conhecimento. Outras pesquisas procuram consolidar características destes atores sociais ou os comportamentos predominantes ao tomarem as decisões, ou mesmo em suas ações empreendedoras dentro de contextos diversos. Este trabalho visou avançar o Effectuation como uma teoria do

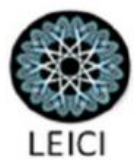


empreendedorismo examinando como ela se relaciona com outros conceitos, modelos e teorias que também buscam compreender e explicar a ação empreendedora, ao tentar entender quais são as ações dos empreendedores que se caracterizam como comportamentos do tipo Causation e Effectuation. As abordagens Causation ou Effectuation e sua opção de utilização pelas organizações não podem ser entendidas como dicotômicas. Assim, ao se referir a uma ou a outra, ambas podem ser entendidas como complementares, concomitantes, ou, ainda, sequenciais ou simultâneas. Sob a concepção da abordagem Effectuation a mesma pode ser uma alternativa para a factibilidade da ação empreendedora, dependente do contexto de imprevisibilidade, das heurísticas cognitivas do empreendedor e, até mesmo, das tomadas de decisão sob dúvida ou incerteza.

Para sua efetividade, sugerimos que pesquisas empíricas trabalhem sob esta lente, abrindo cenários promissores nas organizações e subsidiando resultados que poderiam argumentar que a utilização do Effectuation pelos empreendedores pode ser articulada com a adoção do Causation e como estas estão relacionadas às características das oportunidades empreendedoras, ideias de negócios e processos de desenvolvimento de oportunidades.

\section{Referências}

Alsos, G. A., \& Clausen, T. H. (2014). The start-up processes of tourism firms - the use of causation and effectuation strategies. In G. A. Alsos, D. Eide, \& E. L. Madsen (Eds.), Handbook of Research on Innovation in Tourism. Cheltenham, UK: Edward Elgar. Andersson, S. (2011). International entrepreneurship, born globals and the theory of effectuation. Journal of Small Business and Enterprise Development, 18 (3), 627 - 643. Appelhoff, D., Mauer, R., Collewaert, V., \& Brettel, M. (2015). The conflict potential of the entrepreneur's decision-making style in the entrepreneur-investor relationship. International Entrepreneurship and Management Journal, 1-23.

Blauth, M., Mauer, R., \& Brettel, M. (2014). Fostering Creativity in New Product Development through Entrepreneurial Decision Making. Creativity and Innovation Management, 23(4), 495-509.

Blekman, T. (2011). Corporate effectuation: what managers should learn from entrepreneurs. The Hague: SDU Publishers.

Brettel, M., Mauer, R., Engelen, A., \& Küpper, D. (2012). Corporate effectuation: Entrepreneurial action and its impact on R\&D project performance. Journal of Business Venturing, 27(2), 167-184.

Chandler, G.N., DeTienne, D., McKelvie, A., \& Mumford, A. (2011). Causation and effectuation processes: A validation study. Journal of Business Venturing, 26, 375-390.

Ciszewska-Mlinaric, M.; Obloj, K. \& Wasowska, A. (2016). Effectuation and causation: Two decision-making logics of INVs at the early stage of growth and internationalization.

Journal of East European Management Studies, 275 - 297.

Creswell, J. W. (2009). Research Design: Qualitative, quantitative and mixed methods approaches. 3nd. Thousand Oaks, California: Sage.

Cyert, R. M. \&March, J. (1963). A Behavioral Theory of the Firm. Englewood Cliffs, NJ, v. 2. da Costa, A. F., \& Brettel, M. (2011). Employee effectuation - What makes corporate employees act like entrepreneurs. Frontiers of Entrepreneurship Research, 31(17), 2. 
Davidsson, P. (2004). Researching entrepreneurship. New York: Springer, 2004.

Deligianni, I., Voudouris, I., \& Lioukas, S. (2015). Do Effectuation Processes Shape the Relationship Between Product Diversification and Performance in New Ventures?

Entrepreneurship Theory and Practice.

DeTienne, D. R., \& Chandler, G. N. (2010). The impact of motivation and causation and effectuation approaches on exit strategies. Frontiers of Entrepreneurship Research, 30(1).

Dew, N., Read, S., Sarasvathy, S. D., \& Wiltbank, R. (2009). Effectual versus predictive logics in entrepreneurial decision-making: Differences between experts and novices. Journal of Business Venturing, 24 (4), 287-309.

Dew, N. \& Sarasvathy, S. D. (2007). Innovations, Stakeholders \& Entrepreneurship. Journal of Business Ethics. 74, 267-283.

Engel, Y., Dimitrova, N. G., Khapova, S. N., \& Elfring, T. (2014). Uncertain but able:

Entrepreneurial self-efficacy and novices ' use of expert decision-logic under uncertainty.

Journal of Business Venturing Insights, 1, 12-17.

Evers, N. \& O'Gorman, C. (2011). Improvised internationalization in new ventures: The role of prior knowledge and networks. Entrepreneurship \& Regional Development, 23, 549-574.

Faiez, G. \& Younes, B. (2012). A cognitive approach for analyzing the influence of effectual network on entrepreneurs' actions. In Institute of Interdisciplinary Business Research. 3(9). Fisher, G. (2012). Effectuation, Causation, and Bricolage: A Behavioral Comparison of Emerging Theories in Entrepreneurship Research. Entrepreneurship Theory and Practice, 36(5), 1019-1051.

Filion, L.J. (1991). Vision et relations: clefs du succès de l'entrepreneur, Montreal, Qc: Éditions de l'entrepreneur, $272 \mathrm{p}$.

Filion, L. J. (1999). Empreendedorismo: empreendedores e proprietários-gerentes de pequenos negócios. Revista de Administração, 34 (2), 6-28.

Gabrielsson, J., \& Politis, D. (2011). Career motives and entrepreneurial decision-making: examining preferences for causal and effectual logics in the early stage of new ventures. Small Business Economics, 36(3), 281-298.

Galkina, T. \& Chetty, S. (2015). Effectuation and Networking of Internationalizing SMEs. Management International Review. 55(5) 647-676.

Harms, R \& Schiele, H. (2012). Antecedents and consequences of effectuation and causation in the international new venture creation process. Journal of International Entrepreneurship, 10(1), 95-116.

Kalinic, I., Sarasvathy, S. D., \& Forza, C. (2014). 'Expect the unexpected': Implications of effectual logic on the internationalization process. International Business Review, 23(3), 635647.

Kirzner, I. M. (1979). Perception, opportunity, and profit: Studies in the theory of entrepreneurship. Chicago: University of Chicago Press.

Kirzner, I. M. (1982). The theory of entrepreneurship in economic growth. p. 272-276. In: Kent, D. L.; Sexton, D. L.; Vesper, K. H. (Ed.). Encyclopedia of entrepreneurship. New Jersey: Englewood cliffs.

Knight, F. H. (1921). Risk, uncertainty and profit. New York: Houghton Mifflin. Laine, I \& Galkina, T. (2017). The interplay of effectuation and causation in decision 
making: Russian SMEs under institutional uncertainty. International Entrepreneurship and Management Journal.13: 905.

Laskovaia, A., Shirokova, G. \& Morris, M. (2017). National culture, effectuation, and new venture performance: global evidence from student entrepreneurs. Small Business Economics, 49: 687.

Levinthal, D.A. \& March, J. (1993). The Myopia of Learning. Strategic Management Journal, $14,95-112$.

Long, D., Xia, Z. \& Hu, W. (2017). "How does entrepreneurial opportunity affect the decision-making process of effectuation? Evidence from China", Kybernetes, 46 (6). Machado da Silva, C.L., Cunha, V.C. \& Amboni, N. (1990). Organizações: o estado da arte da produção acadêmica no Brasil. Anais do Encontro da Associação Nacional dos Programas de Pós-Graduação em Administração, 14, Florianópolis, SC, Brasil.

March, J. G. (1991). Exploration e Exploitation in Organizational Learning. Organizational Science, 2(1), 71-87.

March, J. G., \& Olsen, J. P. (1975). Organizational Learning Under Ambiguity. European Journal of Policy Review, 3(2), 147-171.

Mintzberg, H. (1994). The rise and fall of strategic planning. New York: Free Press.

Porter, M. E. (1980). Competitive Strategy: Techniques for analyzing industries and competitors. New York, NY: Free Press.

Read, S.; Dew, N.; Sarasvathy, S. D.; Song, M. \& Wiltbank, R. (2009). Marketing Under Uncertainty: The Logic of an Effectual Approach. Journal of Marketing, 73 (3), 1-18.

Roach, D. C., Ryman, J.A. \& Makani, J. (2016). Effectuation, innovation and performance in SMEs: an empirical study. European Journal of Innovation Management. 19 ( 2), 214-238. Sarasvathy, S. D. (2001a). Causation and Effectuation: Toward a Theoretical Shift from Economic Inevitability to Entrepreneurial Contingency. The Academy of Management Review, 26 (2), 243-263.

Sarasvathy, S. D. (2001b). Effectual reasoning in entrepreneurial decision making: Existence and bounds. Best paper proceedings, Academy of Management, 3-8.

Sarasvathy, S. D. (2008). Effectuation: Elements of entrepreneurial expertise. Cheltenham, UK: Edward Elgar.

Sarasvathy, S.D. \& Dew, N. (2005). New market creation as transformation. Journal of Evolutionary Economics, 15 (5), 533-565.

Sarasvathy, S., Kumar, K., York, J. G., \& Bhagavatula, S. (2014). An effectual approach to international entrepreneurship: overlaps, challenges and provocative possibilities.

Entrepreneurship Theory and Practice, 38(1), 71-93.

Schumpeter, J.A., (1934). The theory of economic development. In: An Inquiry into Profits, Capital, Credit, Interest, and the Business Cycle. Harvard University Press, Cambridge, MA. Shane, S. (2012). Reflections on the 2010 AMR decade Award: Delivering on the Promise of Entrepreneurship as a Field of Research. Academy of Management Review, 37(10), 10-20. Shane, S. A. \& Venkataraman, S. (2000). The promise of entrepreneurship as a field of research. Academy of Management Review, 25, 217 - 226. 
Schweizer, R. (2015). Decision-making during small and medium-sized enterprises internationalization - effectuation vs. causation', J. International Business and Entrepreneurship Development, 8(1), 22-41.

Simon, H. A. (1947). Administrative behavior. New York: Macmillan.

Smolka, K. M., Verheul, I., Burmeister-Lamp, K. \& Heugens, P. P.M.A.R. (2016). Get It Together! Synergistic Effects of Causal and Effectual Decision-Making Logics on Venture Performance. Entrepreneurship Theory and Practice.

Welter, C., Mauer, R., \& Wuebker, R. (2015). Bridging Behavioral Models and Theoretical Concepts: Effectuation and Bricolage in the Opportunity Creation Framework. Forthcoming, Strategic Entrepreneurship Journal Special Issue: Theories of Entrepreneurship.

Sarasvathy Werhahn, D., Mauer, R., Flatten, T. C., \& Brettel, M. (2015). Validating effectual orientation as strategic direction in the corporate context. European Management Journal, 33(5), 305-313.

Wiltbank, R., Read, S., Dew, N., \& Sarasvathy, S. D. (2009). Prediction and control under uncertainty: Outcomes in angel investing. Journal of Business Venturing, 24(2), 116-1 